Original article

\title{
Association of Heart rate variability measured by RR interval from ECG and pulse to pulse interval from Photoplethysmography
}

\author{
Ch Kiran kumar $^{\text {a }}$, M. Manaswini ${ }^{\text {b }}$, K.N. Maruthy ${ }^{\mathrm{c}}$, A.V. Siva Kumar ${ }^{\text {d, }}$, K. Mahesh kumar ${ }^{\mathrm{e}}$ \\ ${ }^{\text {a }}$ Dept. of Physiology, Narayana Medical College and Hospital, Nellore, Andhra Pradesh, India \\ ${ }^{\mathrm{b}}$ Narayana Medical College and Hospital, Nellore, Andhra Pradesh, India \\ ${ }^{\mathrm{c}}$ Dept. of Physiology, Narayana Medical College and Hospital, Nellore, Andhra Pradesh, India \\ ${ }^{\mathrm{d}}$ Faculty \& Ph.D Scholar, Dept. of Physiology, Narayana Medical College and Hospital, Nellore, Andhra Pradesh, India \\ ${ }^{\mathrm{e}}$ Dept. of Physiology \& Biochemistry, Govt. Yoga and Naturopathy College \& Hospital, Chennai, Tamilnadu, 600104, India
}

\section{A R T I C L E I N F O}

\section{Keywords:}

Heart rate variability

Pulse rate variability

Plethysmography

Portable device

\begin{abstract}
A B S T R A C T
Background: Heart rate variability (HRV) is a reliable diagnostic tool for the evaluation of autonomic dysfunction. The pattern of Heart rate reflects on pulse rate. So Pulse rate variability (PRV) is a sensitive indicator of the autonomic tone similar to HRV. The current study aimed to compare PRV measured from pulse plethysmography (PPG) with HRV recorded from ECG.

Materials and Methods: The study was executed in 50 healthy female volunteers aged 18-25 years. The simultaneous recording of lead II ECG and PPG in two channels through audacity software was done for all subjects. Later the R-R interval calculated separately for ECG and Pulse to pulse interval (PRV) from PPG were estimated, and HRV parameters were obtained using Kubios software.

Results: R-R interval mean from ECG $(700 \pm 81.34 \mathrm{msec})$ and PRV $(703.6 \pm 86.28 \mathrm{msec})$ were similar and had a significant positive correlation $(r=0.99)$. Time-domain parameters SDNN $(r=0.61)$, RMSSD $(r=0.78)$, NN50 ( $r=0.790$ and pNN50 ( $r=0.92)$ also has strong positive correlation between the HRV and PRV. Similarly frequency domain, LF Power $(r=0.79)$, HF power $(r=0.75)$, LF in n.u $(r=6.0)$, HF in n.u $(r=5.1)$ also showed very strong correlation. Bland Altman plot reflected a good agreement between the methods for time and frequency domain parameters.

Conclusion: The current study shows that the two methods' values are similar, consistent, and reproducible between HRV and PRV. It suggests PRV can also evaluate sympathovagal balance in different clinical conditions along with HRV in a large community-based study.
\end{abstract}

\section{Introduction}

The Heart rate variability (HRV), by definition, the time interval between the successive heartbeats and the Taskforce of the European Society of Cardiology and the North American Society of Pacing and Electrophysiology, has established the standards two decades ago. ${ }^{1}$ In recent times, HRV provides an index of the parasympathetic nervous system in the heart's specific cardiac vagal tone. ${ }^{2,3}$ Furthermore, HRV represents a simple, economical, and non-invasive measurement, which again draws many scientists in psychophysiology and cardiovascular diseases. In recent times, numerous recording systems exist to measure HRV either through an electrocardiogram (ECG), the interbeat interval (IBI), or Photoplethysmography. ${ }^{4}$ Traditional devices have used ECG recording to estimate $\mathrm{HRV}$ variables due to the high accuracy and more precious in terms of ORS or R peak detection, artifact corrections using electrodes. $^{5,6}$ Though the modern technologies used IBI to measure HRV, solely estimate the time interval between the heartbeats using chest belts or polar heart rate recording devices. These models' significant issues are more artifacts due to the movement against the skin and only depend on the IBI rather than ECG signal or R wave or QRS detection, which lacks accuracy. ${ }^{7}$ Photoplethysmography (PPG) is the technique to record the time serial got from digitalizing the absorbed light measurements, which changes with the periodic blood flow of the artery may utilize as a pulse rate variability (PRV). Previous studies showed an excellent correlation between the HRV and PRV ${ }^{8,9}$; moreover, PRV could be measured very quickly with a smartphone or

\footnotetext{
* Corresponding author. Faculty \& Ph.D scholar Department Of Physiology, Narayana Medical College, Nellore, 524003, Andhra Pradesh, India.

E-mail addresses: kiranchintala26@gmail.com (C. Kiran kumar), mullagirimanaswini@gmail.com (M. Manaswini), Maruthy.dr@gmail.com (K.N. Maruthy), reddy.sivakumar5@gmail.com (A.V. Siva Kumar), doctormahesh1985@gmail.com (K. Mahesh kumar).
} 


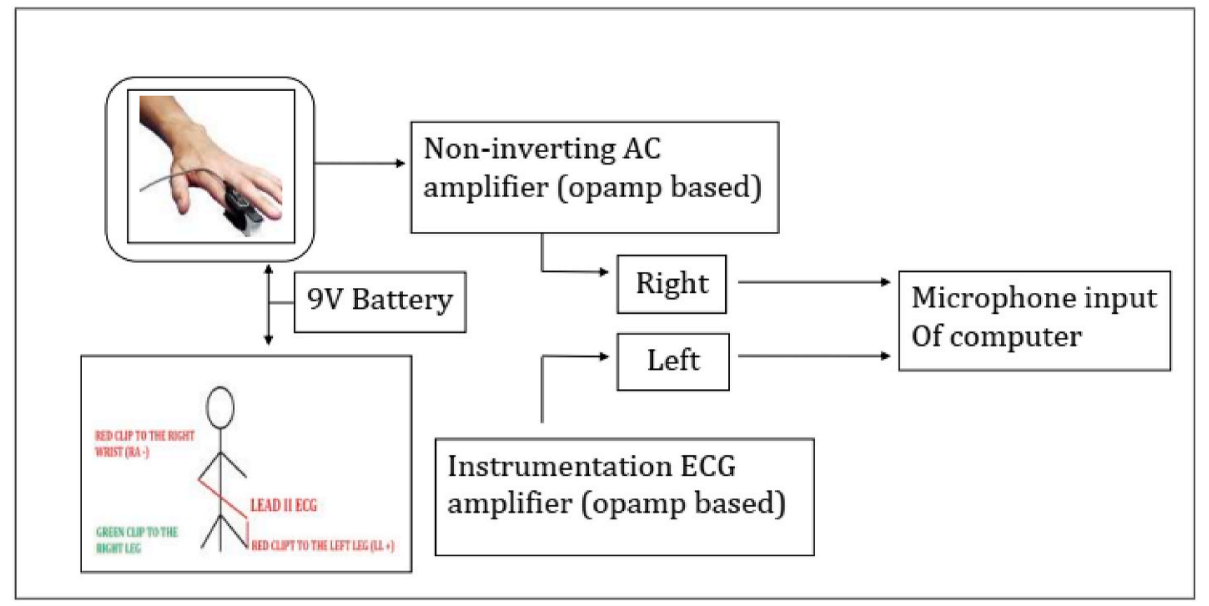

Fig-1. shows the block diagram of synchronous RR and PP interval data acquisition.

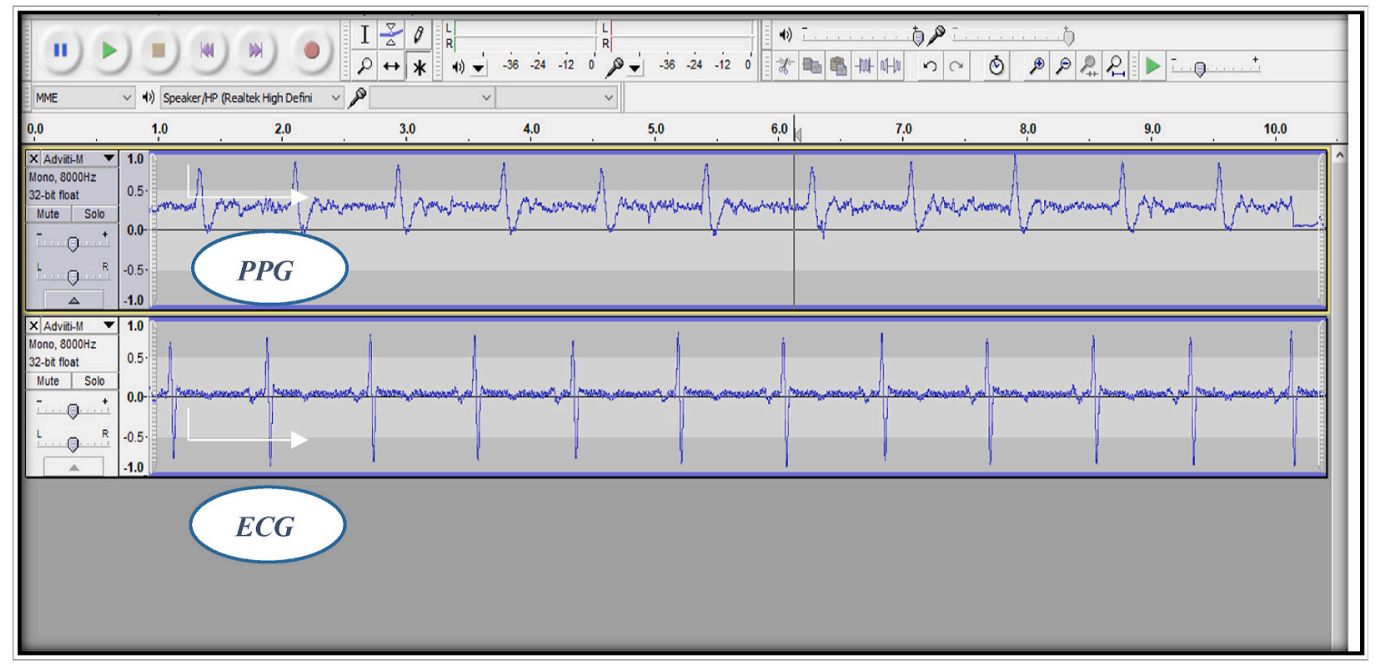

Fig. 2. Shows the synchronized recording of ECG and PPG in dual channel of Audacity software.

Table 1

Demographic parameters of the subjects.

\begin{tabular}{llll}
\hline Age $(\mathrm{yrs})$ & $20.19 \pm 1.62$ & 19.78 & $19.25-21.54$ \\
\hline Weight $(\mathrm{kgs})$ & $62.23 \pm 11.56$ & 60 & $57.56-66.9$ \\
Height $(\mathrm{cms})$ & $155.8 \pm 11.77$ & 159 & $152.7-163$ \\
BMI $\left(\mathrm{kg} / \mathrm{m}^{2}\right)$ & $25 \pm 4.1$ & 24.05 & $23.35-26.67$ \\
Variables & Mean $\pm \mathrm{SD}$ & Median & $95 \% \mathrm{CI}$ \\
\hline
\end{tabular}

low-cost tool by anyone. ${ }^{10,11}$ In recent decades, progress in optoelectronics and digital signal processing advancements have facilitated a renaissance of PPG, which today is probably the most popular method used in clinical monitoring. PPG technology is that it is non-invasive, cost-effective, and straightforward to use. ${ }^{12}$ Compared to the current standard of an ECG-based HRV analysis, this would involve certain benefits. In clinical situations where a pulse oximeter (PO) device is already at hand by default, including HRV analysis in the monitoring process without requiring an ECG means a significant advantage. Besides magnetic resonance imaging (MRI), ECG electrodes or other metal-containing sensors are not permitted, as they interfere with strong electromagnetic fields. Detecting the PPG signal usually requires no more than attaching a single sensor to a finger or an earlobe, compared to at least three leads and electrodes are necessary for an ECG. These electrodes often have to be applied to the chest, requiring the patients to undress, delaying recordings, and pose a problem for embarrassed patients. ${ }^{13}$ Most of these ECG and PPG instruments are expensive and unaffordable to the routine clinical setups. We designed and developed a PPG apparatus (transmission type) with A to D converter to obtain finger pulse plethysmography (PPG) through which PRV is analyzed. So the current study was aimed at the validation of PRV obtained from PPG compared with HRV obtained from ECG to assess the reliability and accuracy of time and frequency domain parameters that reflect the autonomic balance.

\section{Materials and Methods}

\subsection{Study population}

The present was included 50 healthy volunteers of female medical students aged between 18 and 25 years. Being a pilot study sample size was not calculated. The study commenced after getting ethical clearance from the host Institutional Ethics committee. Apparent healthy subjects were included based on the casual inquiry of previous medical illness, recent medication, if any, and other routine activities of daily life. The participants with abnormal menstrual cycles were excluded from the study. However, all of them are medical students, so it becomes easier to evaluate their health status with a casual inquiry. Detailed information about the study's nature and purpose was explained to the participants 
Table 2

Comparison of time domain and frequency domain parameters of ECG and PPG. SDNN ( $r=0.61)$, RMSSD (0.78), NN50(0.790 and pNN50 (0.92).

\begin{tabular}{|c|c|c|c|c|}
\hline \multirow[t]{2}{*}{ Variable } & \multicolumn{2}{|l|}{ Sitting } & \multirow{2}{*}{$\begin{array}{l}\text { Correlation } \\
\text { coefficient (r) }\end{array}$} & \multirow[t]{2}{*}{$95 \% \mathrm{CI}$} \\
\hline & ECG & PPG & & \\
\hline \multicolumn{5}{|l|}{ Time domain } \\
\hline $\begin{array}{l}\text { Mean RR } \\
\quad(\mathrm{ms})\end{array}$ & $\begin{array}{l}700.5 \pm \\
81.34\end{array}$ & $\begin{array}{l}703.6 \pm \\
86.28\end{array}$ & 0.99 & $0.98-0.99$ \\
\hline $\begin{array}{c}\text { Mean HR } \\
\text { (Beats) }\end{array}$ & $87.3 \pm 9.6$ & $\begin{array}{l}87.5 \pm \\
11.11\end{array}$ & 0.96 & $0.93-0.98$ \\
\hline SDNN (ms) & $\begin{array}{l}53.95 \pm \\
16.62\end{array}$ & $\begin{array}{l}61.18 \pm \\
29.24\end{array}$ & 0.61 & $0.38-0.76$ \\
\hline RMSSD (ms) & $\begin{array}{l}39.04 \pm \\
16.32\end{array}$ & $\begin{array}{l}35.80 \pm \\
20.13\end{array}$ & 0.78 & $0.61-0.86$ \\
\hline NN50 & $\begin{array}{l}55.20 \pm \\
46.02\end{array}$ & $\begin{array}{l}61.80 \pm \\
49.24\end{array}$ & 0.79 & $0.64-0.88$ \\
\hline pNN $50(\%)$ & $\begin{array}{l}14.99 \pm \\
13.7\end{array}$ & $\begin{array}{l}16.73 \pm \\
13.85\end{array}$ & 0.92 & $0.86-0.96$ \\
\hline \multicolumn{5}{|c|}{ Frequency Domain } \\
\hline $\begin{array}{l}\text { LF Power } \\
\text { (ms2) }\end{array}$ & $\begin{array}{l}1128.0 \pm \\
702.4\end{array}$ & $\begin{array}{l}1057.2 \pm \\
683.1\end{array}$ & 0.79 & $0.71-0.93$ \\
\hline $\begin{array}{l}\text { HF Power } \\
\text { (ms2) }\end{array}$ & $\begin{array}{l}658.3 \pm \\
575.1\end{array}$ & $\begin{array}{l}834.1 \pm \\
752.2\end{array}$ & 0.75 & $0.69-0.94$ \\
\hline LF nu & $\begin{array}{l}65.18 \pm \\
13.91\end{array}$ & $\begin{array}{l}59.69 \pm \\
13.56\end{array}$ & 0.60 & $0.35-0.70$ \\
\hline HF nu & $\begin{array}{l}34.61 \pm \\
13.88\end{array}$ & $\begin{array}{l}40.12 \pm \\
13.54\end{array}$ & 0.51 & $0.25-0.70$ \\
\hline LF/HF & $1.87 \pm 1.83$ & $1.68 \pm 0.85$ & 0.80 & $0.75-0.86$ \\
\hline
\end{tabular}

and obtained informed consent before study enrollment as per the Declaration of Helsinki 1975. All of them were instructed to abstain caffeinated beverages before the day of recording and not perform any physical exercise on the day of data collection. Height and weight were measured, and a stadiometer and electronic weighing machine. The subjects were allowed to rest in the sitting posture for $10 \mathrm{~min}$ before initiation of the protocol. Later lead II ECG, and PPG signals were obtained simultaneously from each subject in the morning after light breakfast in the Research lab of the Department of Physiology of the host institute.

\subsection{ECG device}

After 10 min of rest, Lead II ECG recorded using the Analog ECG amplifier with A to D (Analogue to Digital) conversion using the computer's sound card. The RR interval was extracted and analyzed using KUBIOS 1.1 software, Finland, used to measure the different variables such as the time or frequency domain. Time-domain analysis calculated directly from the raw RR-interval. The frequency-domain shows the RRsignal variability over time by looking at the spectra's proportion relative to the original RR-signal. Frequently used time-domain parameters are mean and standard deviation (SD) of RR, NN50 (number of consecutive RR intervals that differ more than $50 \mathrm{~ms}$ ), and pNN50 (proportion of NN50). Frequently used spectral measures are peak frequency and power of very low-frequency bands (VLF), low-frequency bands (LF), high-frequency bands (HF), and LF/HF ratio. ${ }^{14}$

\subsection{Photo pulse plethysmography (PPG)}

The Finger photo pulse plethysmography uses an LED-based Infrared (IR) transmitter and receiver that detects the finger pulses in transmittance mode. It was an in-house built device in which the PPG sensor used low-cost optoelectronic components operating in the domain of infrared wavelengths with $5 \mathrm{~mm}$ LED's, and the intensity of light was $5 \mathrm{~mW}$. The signals obtained from the IR receiver amplified using a noninverting "op-amp" based AC amplifier. The increased output is connected to the microphone input of the PC sound card. ${ }^{24}$ Changes arise PPG signal arises in the path and length between source and optical density of blood. The signal can decompose into two parts. The small pulsatile component, or AC component, arises from arterial blood pulsation; hence its oscillation parallels momentary cardiac activity. It superimposes on the much more significant DC component, Where DC refers to direct current, suggesting a static behavior. However, the DC component is not entirely static but included variations slower than the heart rate due to venous volume fluctuations and thermoregulation (Fig. 1).
Bland-Altman of RR interval

a)

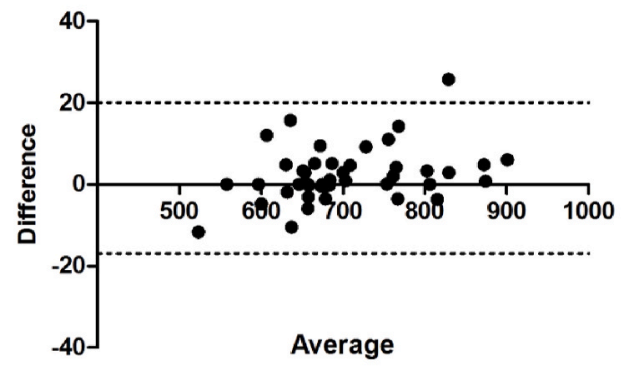

c)

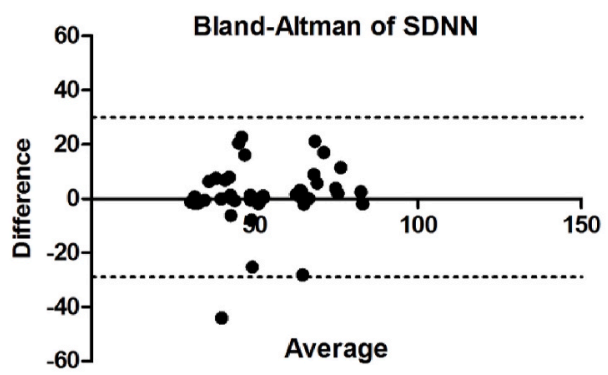

b)

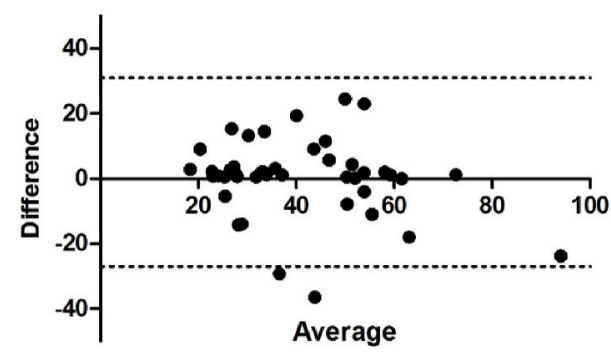

Bland-Altman of pNN50

d)

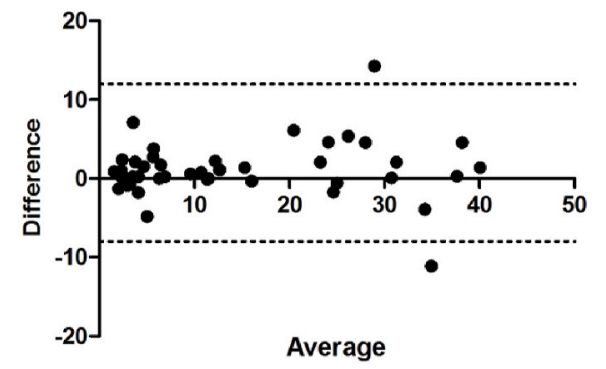

Fig. 3. Bland-Altman plots of time-domain indexes, i.e., (a) RR interval, (b) RMSSD, (c)SDNN, and (d) pNN50 showing mean values of EEG -based and PPG based indexes against their difference. For each figure, Horizontal lines denote median (solid lines) and 95\% confidence intervals of the difference PPI-RRI (dotted lines). 

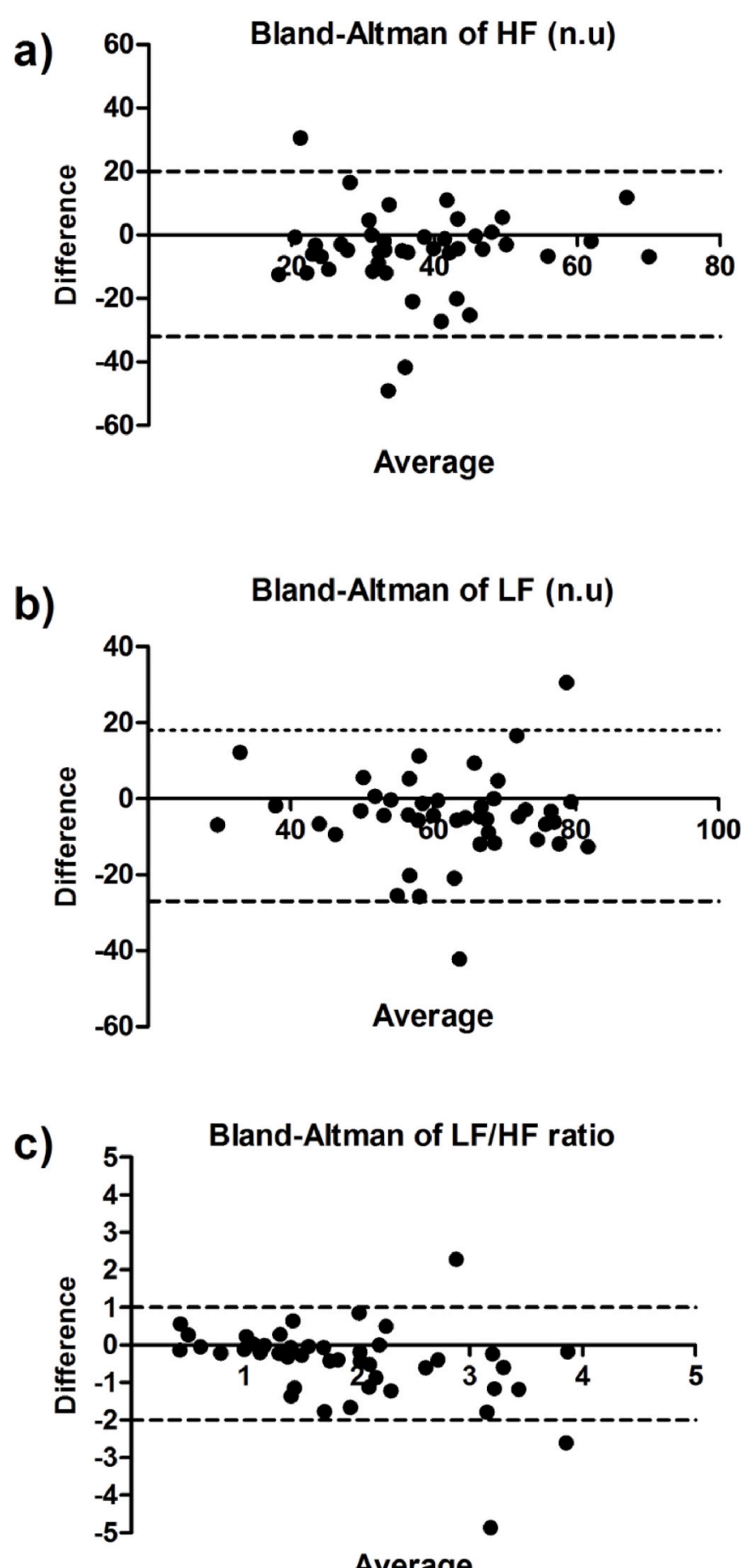

Fig. 4. Bland-Altman plots of frequncey-domain indexes, i.e., (a) HF (n.u), (b) LF (n.u), and (c) LF/HR ratio showing mean values of EEG -based and PPG based indexes against their difference. For each figure, Horizontal lines denote median (solid lines) and 95\% confidence intervals of the difference PPI-RRI (dotted lines).

\subsection{Audacity}

Audacity is sound recording software that is freely available in the study to record and display the real-time ECG and PPG recordings. It acts as an A/D converter, and an advantage to using the computer's sound card as an A/D converter was that it eliminates the additional requirement of an external microprocessor. Sound editing software can display real-time signals with time and amplitude analysis solutions. Simple
ECG and PPG analog amplifiers are used to acquire the synchronizing ECG and PPG signals. The digitalization of the analog signals done using the sound card of a computer (laptop). The display of the recording was done by using Audacity sound editing software (version 1.2.2) in wave format. This Audacity software has many offline editing options for obtaining the RR interval and PP interval in a simple manner. Electrical noise (50) $\mathrm{Hz}$ in the digital data was filtered using a low pass filter. $\mathrm{R}$ waves identified using the beat to beat the finder tool in the audacity software by fixing the amplitude as $60 \mathrm{~Hz}$. If the waves' magnitude was low, it could increase to a desirable amplitude using the amplify option (Fig. 2). Later, R peak, which was identified by beat finder, was converted into real-time RR interval data by using exporting the labels option in the software, and it has stored in the note pad format. ${ }^{15}$

\subsection{Statistical analysis}

Continuous variables are expressed as mean \pm standard deviation. The Man - Whitney $U$ test was used to compare the differences between measurements. We assessed the agreement between the devices using the Bland-Altman methodology and the Spearman correlation analysis to determine correlation coefficient (CC) and coefficient of variation. Statistical significance was set as $\mathrm{p}<0.05$.

\section{Results}

Table 1 shows the demographic variables of the subjects. The mean age of the participants is $20.19 \pm 1.62$ years $(\mathrm{CI}=19.25-21.54)$. There was no significant difference between the variables of the two devices. All variables have a p-value $>0.05$. Table 2 showed correlation results for the features obtained from PPG and ECG of the subjects. As shown in Table 2, Time-domain parameters (NN intervals, RMSSD, SDNN, NN50, and pNN50) and frequency parameters demonstrated a strong correlation $(r>0.90, p<0.05)$ between the ECG and PPG. Regarding the comparison between the two approaches to compute the same index in a given subject, we observed that the distributions of the mean index of HRV parameters were statistically $(\mathrm{P}>0.05)$ similar. The Bland-Altman analysis of all features computed from ECG and PPG represents a close agreement for all the parameters (see Fig. 3 and 4).

\section{Discussion}

The current study finding suggests a good agreement between HRV variables measured from the R-R interval by ECG and pulse-to-pulse interval variability (PRV) by PPG. The previous findings Gil E et al., 2010, Heathers JA et al., 2013, and Hayano et al., 2005 also had a strong correlation for PRV and HRV. It confirmed PRV as a reliable alternate of HRV, which has been used for avoiding the confounding factor of respiration in HRV evaluation. ${ }^{16-19}$ However, few studies like Constant I et al., 1999, have opposed the agreement mentioned above ${ }^{20}$ It can also be affected by physical activity, mental stress, hemodynamic alterations, or metabolic changes. In addition to this, HRV has some other problems like adhesive electrode patches, complex morphology, drift, and many wires. The PPG method used the intelligent optical sensors that are robust and need less maintenance, and it is ideal for a mobile device. Moreover, the PPG signal can be used for clinically relevant parameters like heart and respiratory rate and respirational induced intensity variations. In recent development and advancements in digital signal processing, PPG usage in clinical monitoring has been increased in practice and research. Modern PPG sensors use low-cost optoelectronic modules working with the red or infrared wavelengths. It is also used to estimate pulse wave velocity, cardiac output, peripheral arterial occlusion, endothelial and venous function, etc.

To summarize the Bland-Altman method's most critical analysis, we collected the SD (standard deviation) and BAR (Bland-Altman ratio) values for the various HRV indices corresponding to the ECG-PPG comparisons. We found excellent agreement between the indices of 
both methods.

Wong, Jih-Sen et al. 2012 found that PRV exhibited highly significant coherence between all HRV measures and the corresponding PRV measures of both hands. ${ }^{21}$ So we have been used the left side for a better and reliable assessment and found acceptable agreement between the PRV and HRV. In contrast, others have found that no appreciable agreement was found between PRV and HRV. Numerous factors might be involved in the difference between HRV and PRV in many conditions. Electrical waves originated from the heart in HRV are different from the mechanical waves produced in vessels for PRV by physical nature.

Along with that, the caliber of the blood vessels, the viscosity and osmolarity of the blood, the autonomic nervous modulation, and the subjects' body frame might change the PRV and contribute to the variation. Likewise, electrodes' application onto the chest wall and beneath the breasts is generally inconvenient for participants for recording ECG signals for HRV in a large community-based study. For PRV, just a single probe placed to either hand the subject's finger can act as the surrogate of HRV, and without having to undress a participant. Thus, however, it is a regular practice to acquire HRV from the R-R intervals. There have been efforts to practice peripheral pulse wave signals to assess autonomic nervous modulation for community-based research and screening. The present study has some limitations; this study is only executed in females adolescents who are acquaintances to the investigator. This protocol will be extrapolated to the large sample of healthy volunteers with both genders and different groups of patients to characterize the study results.

\subsection{Clinical applications of $H R V \& P R V$}

The HRV and PRV is early indicator of ANS dysfunction which helps to evaluate primary or secondary autonomic failures. In addition it also helps to reduce mortality and morbidity associated with cardiovascular disorders. ${ }^{22,23}$ They are user friendly, noninvasive tools in sports evaluation, exercise interventional studies and prognosis in other neurological diseases. ${ }^{25}$

\section{Conclusion}

The current study reports suggest PRV can also be used to evaluate cardiac autonomic tone. It is cost-effective; the portable, user-friendly device provides reliable readings to evaluate autonomic dysfunction quantitatively. Practitioners in the community field studies can use this device.

\section{Funding}

There is no financial support for this research work and publication of the article.

\section{Declaration of competing interest}

On behalf of all the authors, the corresponding author states no potential conflict of interest regarding the research work and publication.

\section{Acknowledgment}

The authors would like to express their sincere gratitude to Narayana Medical College and Hospital's participants and management for their constant support and encouragement throughout the study period.

\section{References}

1 Malik M. Heart rate variability: standards of measurement, physiological interpretation, and clinical use: Taskforce of the European society of Cardiology and the North American society for pacing and Electrophysiology. Ann Noninvasive Electrocardiol. 1996 Apr;1(2):151-181.

2 Laborde S, Mosley E, Thayer JF. Heart rate variability and cardiac vagal tone in psychophysiological research-recommendations for experiment planning, data analysis, and data reporting. Front Psychol. 2017 Feb 20;8:213.

$3 \mathrm{Li} \mathrm{Z}$, Snieder H, Su S, et al. A longitudinal study in youth of heart rate variability at rest and in response to stress. Int J Psychophysiol. 2009 Sep 1;73(3):212-217.

4 Schäfer A, Vagedes J. How accurate is pulse rate variability as an estimate of heart rate variability?: a review on studies comparing photoplethysmographic technology with an electrocardiogram. Int J Cardiol. 2013 Jun 5;166(1):15-29.

5 Kleiger RE, Stein PK, Bigger Jr JT. Heart rate variability: measurement and clinical utility. Ann Noninvasive Electrocardiol. 2005 Jan;10(1):88-101.

6 Munoz ML, van Roon A, Riese H, et al. Validity of (ultra-) short recordings for heart rate variability measurements. PloS One. 2015;10(9).

7 Jarrin DC, McGrath JJ, Giovanniello S, Poirier P, Lambert M. Measurement fidelity of heart rate variability signal processing: the devil is in the details. Int J Psychophysiol. 2012 Oct 1;86(1):88-97.

8 Leikan GM, Rossi E, Sanz M, et al. Evaluation of agreement between temporal series obtained from electrocardiogram and pulse wave. J Phys Conf Ser. 2016 Apr;705(1), 012038. IOP Publishing.

9 Peng R-C, Zhou X-L, Lin W-H, Zhang Y-T. Extraction of heart rate variability from smartphone photoplethysmograms. Comput Math Methods Med. 2015;2015:1-11. https://doi.org/10.1155/2015/516826, 11 pages, 2015, Article ID 516826.

10 Cho Y, Julier SJ, Bianchi-Berthouze N. Instant stress: detection of perceived mental stress through smartphone photoplethysmography and thermal imaging. JMIR Mental Health. 2019;6(4), e10140.

11 Heathers JA. Smartphone-enabled pulse rate variability: an alternative methodology for the collection of heart rate variability in psychophysiological research. Int $J$ Psychophysiol. 2013 Sep 1;89(3):297-304.

12 Schäfer A, Vagedes J. How accurate is pulse rate variability as an estimate of heart rate variability?: a review on studies comparing photoplethysmographic technology with an electrocardiogram. Int J Cardiol. 2013 Jun 5;166(1):15-29.

13 Bánhalmi A, Borbás J, Fidrich M, Bilicki V, Gingl Z, Rudas L. Analysis of a pulse rate variability measurement using a smartphone camera. J Healthc Eng. 2018, 2018.

14 Maheshkumar K, Dilara K, Maruthy KN, Sundareswaren L. Validation of PC-based sound card with biopac for digitalization of ECG recording in short-term HRV analysis. N Am J Med Sci. 2016 Jul;8(7):307.

15 Pullaganti M, Kumar SA, Maruthy KN, Gurja JP, Chintala KK. Association of stress with heart rate variability in different phases of the menstrual cycle. Natl J Physiol Pharm Pharmacol. 2019;9(3):256-260.

16 Gil E, Orini M, Bailón R, Vergara JM, Mainardi L, Laguna P. Photoplethysmography pulse rate variability as a surrogate measurement of heart rate variability during non-stationary conditions. Physiol Meas. 2010 Aug 11;31(9):1271.

17 Heathers JA. Smartphone-enabled pulse rate variability: an alternative methodology for the collection of heart rate variability in psychophysiological research. Int $J$ Psychophysiol. 2013 Sep 1;89(3):297-304.

18 Sun Y, Hu S, Azorin-Peris V, Kalawsky R, Greenwald SE. Noncontact imaging photoplethysmography to effectively access pulse rate variability. J Biomed Optic. 2012 Oct;18(6), 061205.

19 Hayano J, Barros AK, Kamiya A, Ohte N, Yasuma F. Assessment of pulse rate variability by the method of pulse frequency demodulation. Biomed Eng Online. 2005 Dec; 4(1):62.

20 Constant I, Laude D, Murat I, Elghozi JL. Pulse rate variability is not a surrogate for heart rate variability. Clin Sci. 1999 Oct 1;97(4):391-397.

21 Wong JS, Lu WA, Wu KT, Liu M, Chen GY, Kuo CD. A comparative study of pulse rate variability and heart rate variability in healthy subjects. J Clin Monit Comput. 2012 Apr 1;26(2):107-114.

22 Kumar CK, Kumar AS, Madhurima P, Maruthy KN, Preetham GJ. Assessment of psychomotor skills using finger pulse guided biofeedback tool in young medical students: psychomotor skills using heart rate as biofeedback tool. Ann Med Physiol. 2018 Dec 31;2(4):36-39.

23 Ramadevi P, Maruthy KN. Evaluation of static pupil size using digital web camera through image analysis. Int J Physiol. 2019 Jul 25;7(3):7-11.

24 MaheshKumar K, Maruthy KN, Padmavathi R. Comparision of photo pulse plethysmography module with Mobil-O-graph for measurement of pulse wave velocity. Clin Epidemiol Glob Health. 2020 Sep 25;9:216-220. https://doi.org/ 10.1016/j.cegh.2020.09.001.

25 Maheshkumar K, Sivakumar AV, Maruthy KN, Padmavathi R. Hot arm and foot bath on heart rate variability and blood pressure in healthy volunteers-needs to be verified with standard device? J Compl Integr Med. 2020 Sep 9:1 (ahead-of-print). 